\title{
Forest Fire Finder - DOAS application to long-range forest fire detection
}

\author{
Rui Valente de Almeida ${ }^{1,2}$ and Pedro Vieira ${ }^{1,2}$ \\ ${ }^{1}$ NGNS, Ingenious Solutions, Rua Cidade de Évora, 11, 2660-022 Loures, Portugal \\ ${ }^{2}$ Faculty of Science and Technology, NOVA University of Lisbon, Caparica Campus, 2829-516 Caparica, Portugal \\ Correspondence to: Pedro Vieira (pedro.vieira@ngns-is.com)
}

Received: 23 September 2016 - Discussion started: 14 November 2016

Revised: 11 April 2017 - Accepted: 8 May 2017 - Published: 22 June 2017

\begin{abstract}
Fires are an important factor in shaping Earth's ecosystems. Plant and animal life, in almost every land habitat, are at least partially dependent on the effects of fire. However, their destructive force, which has often proven uncontrollable, is one of our greatest concerns, effectively resulting in several policies in the most important industrialised regions of the globe.

This paper aims to comprehensively characterise the Forest Fire Finder (FFF), a forest fire detection system based mainly upon a spectroscopic technique called differential optical absorption spectroscopy (DOAS). The system is designed and configured with the goal of detecting higher-thanthe-horizon smoke columns by measuring and comparing scattered sunlight spectra. The article covers hardware and software, as well as their interactions and specific algorithms for day mode operation. An analysis of data retrieved from several installations deployed in the course of the last 5 years is also presented.

Finally, this paper features a discussion on the most prominent future improvements planned for the system, as well as its ramifications and adaptations, such as a thermal imaging system for short-range fire seeking or environmental quality control.
\end{abstract}

\section{Introduction}

Fire is a process by which elements chemically combine with oxygen, releasing energy (as heat and light) and smoke into the surrounding environment. Fires are an important factor in shaping Earth's ecosystems. Plant and animal life, in many land habitats, are at least partially dependent on the effects of fire (Food and Agriculture Organisation, FAO).

The use of fire by hominids predates civilisation by thousands of years and, in today's society, there are almost no areas of technology or scientific knowledge that do not involve fire in one way or another. However, fire's destructive power is undeniable.

Forest fires are among the great concerns of the present day in industrialised countries. Research regarding wildfires has been targeted by many countries and unions worldwide in an effort to minimise the negative impact these events imply.

According to the Intergovernmental Panel on Climate Change (IPCC), climate change is expected to increase global temperatures and change rainfall patterns, leading to an increased risk of fire (IPCC, 2012). This means that the number of registered fires throughout the world is expected to increase, a phenomenon the world must be ready to address.

In the European Union, the Horizon2020 research programme states that there must be a union-wide investment in research concerning forest protection and recovery from fires. In the past, the FP7 programme had sponsored the development of an automatic forest fire detection system called FireSense, an investment of over EUR 2.5 million (European Comission, 2012).

The United States Forest Service acknowledge the importance of understanding wildland fire dynamics, running a network of research centres solely dedicated to the study of this subject. Research endeavours take $6 \%$ of the service's annual budget, which is currently directed primarily towards fire suppression (United States Forest Department, 2015). 
Australia is another geographic region where wildfires have had a great impact. As a response, its government has created the Bushfire and Natural Hazards Cooperative Research Centre. The institution builds upon more than 10 years of experience dealing with Australian bushfires and aims to produce internationally recognised research regarding the study and modelling of wildfires in Australia and New Zealand (BNHCRC, 2016).

In spite of this global investigation effort regarding fires and their behaviour, every year, material losses as a result of fires ascend to billions of dollars and thousands of lives are lost in the same way. This leads to a strong increase in the size of the fire protection market, including passive and active detection platforms, which is expected to grow at a cumulative aggregate growth rate of $11.53 \%$ from 2014 to 2020 (Research and Markets, 2016).

\section{State of the art}

In recent years, several methods have been developed in an attempt to automatically and reliably detect forest fires. These systems differ primarily in their strategic approach to the issue at hand, creating three main categories:

- Satellite monitoring techniques: satellite data have been used for fire monitoring purposes since the late 20th century. The MODIS (MODerate resolution Imaging Spectroradiometer) and AVHRR (Advanced Very High Resolution Radiometer) sensors, deployed respectively in the Aqua/Terra and NOAA satellites, have had extensive use in this regard. However, their low temporal resolution ( 2 and 4 times per $24 \mathrm{~h}$, respectively) make them poor candidates for fire detection uses. Geostationary satellites overcome this difficulty by continuously scanning a single, very large geographic region. They have, nevertheless, a low spatial resolution of $1 \mathrm{~km}$, which means that small fires are difficult for them to detect (Manyangadze, 2009).

- Wireless network sensing: the wireless sensor network approach to fire detection is completely different from the other two categories. Instead of having a single device patrolling the target region, these systems are designed on the capabilities of a high number of extremely small battery-operated sensor boards that can communicate among themselves (Alkhatib, 2014; Liyang et al., 2005).

The sensor boards are equipped with several sensors, from temperature and humidity to luminance detectors. In spite of their great fire detection capabilities, these networks present various drawbacks, such as their very limited individual range of detection and their 2-year lifetime or the fact that their remains might imply an environmental issue (Alkhatib, 2014).
- Large-area remote sensing: this family of systems is designed with the goal of minimising the number of deployed devices in a given target region. Their architecture implies the use of an optical principle in order to detect smoke or flames, whether optical cameras or spectrometers.

There are already several commercially available systems, such as the Forest Fire Finder (FFF; the main subject of this paper), FireWatch, ForestWatch, AlarmEYE or Eyefi SPARC. Although these are commercial products, the available information is sparse and many times outdated, so a true comparison not only is beyond the scope of the article but also would require more research efforts. Nevertheless, it is important to briefly describe the operating principles of the more prominent systems.

- ForestWatch, developed in South Africa by EnviroVision Solutions, uses optical object recognition software, coupled to a very specific camera system. It detects smoke during the day and the flame glow during the night, at a maximum distance of $24 \mathrm{~km}$ in every direction, in a semi-automatic fashion. It is probably the most commercially successful system, with more than 300 currently operating towers (Envirovision Solutions, 2015; Hough, 2007).

- FireWatch is a commercial system operated and sold by IQ Wireless Gmbh, in Germany. The system uses optical sensors and object recognition algorithms to detect smoke at a maximum distance of $15 \mathrm{~km}$. It is important to mention that the FireWatch system is not a fully automatic fire detection platform, requiring a control room to operate correctly (IQ-Wireless, 2016).

- The FFF was developed in Lisbon, in a partnership between the NOVA University of Lisbon and NGNS-IS, Ltd., in 2006. This patented system uses a spectroscopic technique to assess the atmosphere and detect smoke columns (NGNS-IS, 2016). During the night, the system changes its operation mode and relies solely on image processing to detect a fire's glow. Its maximum rate detection range is of $15 \mathrm{~km}$, and it acts with complete autonomy, requiring minimal human intervention (see Fig. 1).

The FFF's most significant advantage over its rivals is its low number of false alarms (typically one or two per week). This comes from the fact that the system's smoke-detection capabilities do not rely on image processing. This in turn means that reliable detections can be achieved by a smaller number of deployed devices (only one, three for triangulation). However, more reliable alarms imply less human intervention, which translates into less financial expenditure over time.

The FFF system is the only one to use an optical spectroscopy technique to detect fire through smoke presence in 


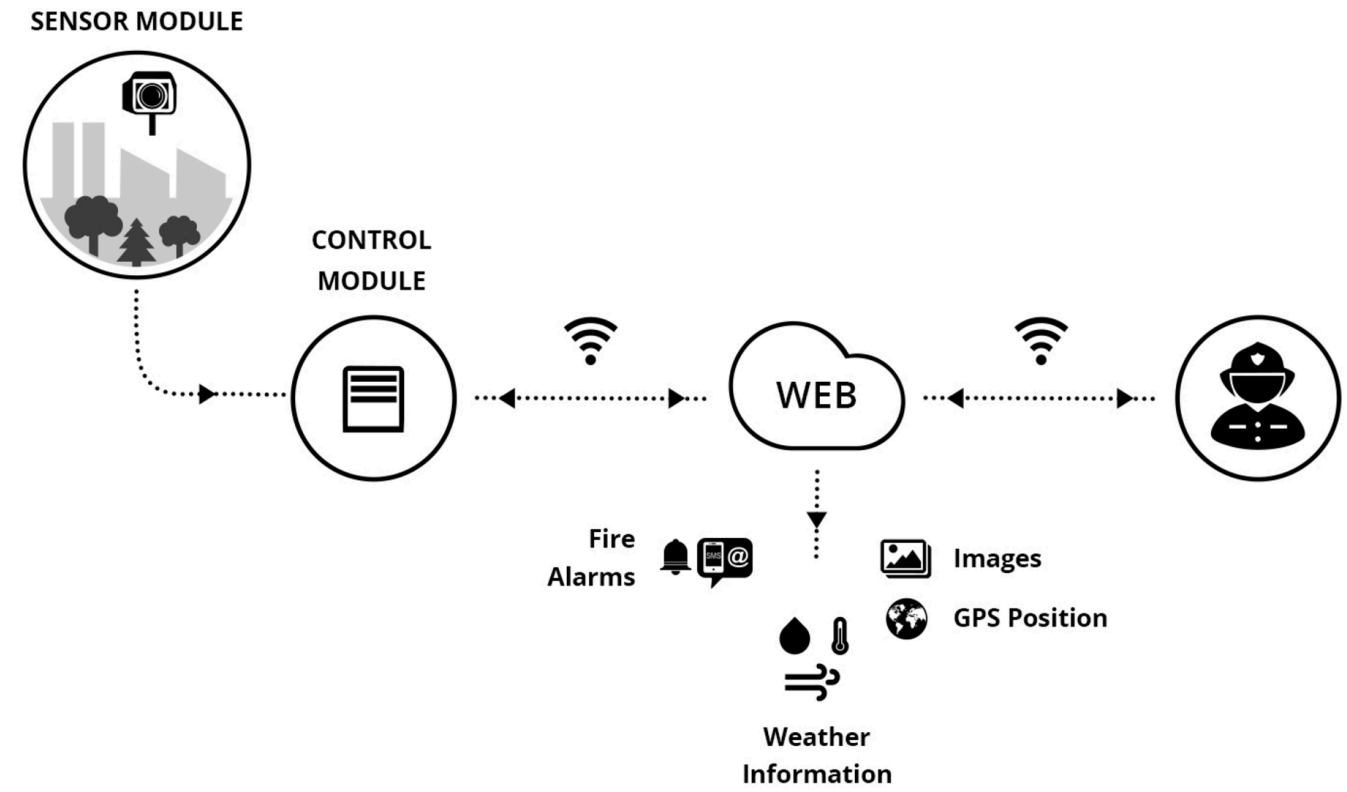

ร Wireless, GSM/GPRS, 3G/UMTS, ADSL, Satellite, Radio, etc...

Figure 1. The Forest Fire Finder system (NGNS-IS, 2016).

real time. Since the analysis is carried out in an outdoor scenario, the process is not as straightforward as in laboratory experiments. This article addresses only the spectroscopic techniques used in the system's daytime operation mode.

\section{The technique}

The FFF system makes use of a spectroscopic technique called differential optical absorption spectroscopy (DOAS). This is a well-established and widely used technique in the field of atmospheric studies (Platt and Stutz, 2007).

There are two main categories of DOAS experiment assemblies, with different goals and capabilities:

- Active systems, of which a simple illustration is presented in Fig. 2, are characterised by relying on an artificial light source for their measurements. A spectrometer at the end of the light path performs spectroscopic detection. Active DOAS techniques are very similar to traditional in-lab absorption spectroscopy techniques (Platt and Stutz, 2007);

- Passive DOAS techniques, illustrated in Fig. 3, use natural light sources, such as the Sun and the moon, in their measurement process. An optical system is pointed in certain elevation and azimuth angles and sends the captured light into a spectrometer, connected to a computer. The system returns the total value of the light absorption in its path (Platt and Stutz, 2007; Merlaud, 2013). Since the FFF system is basically a passive DOAS sys- tem, we will centre our discussion on this category from this point forward.

DOAS itself is based on Lambert-Beer's law, which can be written as (Platt and Stutz, 2007)

$I(\lambda)=I_{0}(\lambda) \cdot \exp (-\sigma(\lambda) \cdot c \cdot L)$,

where $\lambda$ is the wavelength of the emitted light; $I(\lambda)$ is the light intensity as measured by the system; $I_{0}(\lambda)$ is the intensity of the light as emitted by the source; and $\sigma(\lambda)$ is the absorption cross section of absorber, which is wavelength dependent; $c$ is the concentration of the absorber we want to measure.

This law allows the definition of optical thickness $(\tau)$ (Platt and Stutz, 2007):

$\tau(\lambda)=\ln \left(\frac{I_{0}(\lambda)}{I(\lambda)}\right)=\sigma(\lambda) \cdot c \cdot L$.

In a laboratory setting, Eq. (1) or (2) can be used to directly calculate an absorber's concentration, provided there is knowledge of its cross section. In the open atmosphere, however, absorption spectroscopy techniques are far more complex. On one hand, $I_{0}(\lambda)$ is not accessible since we measure from inside the medium we want to measure. On the other hand, there are several environmental and instrumental effects that influence measurement results. These effects include the following (Platt and Stutz, 2007).

- Rayleigh scattering is due to small molecules present in the atmosphere and is heavily influenced by wavelength (hence the blue colour of the sky). 


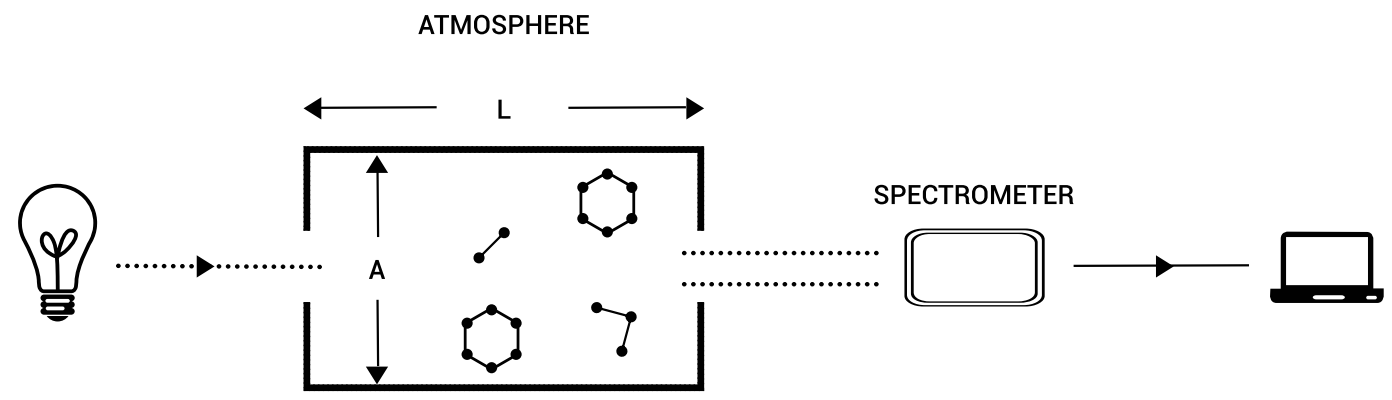

Figure 2. Active DOAS schematic.

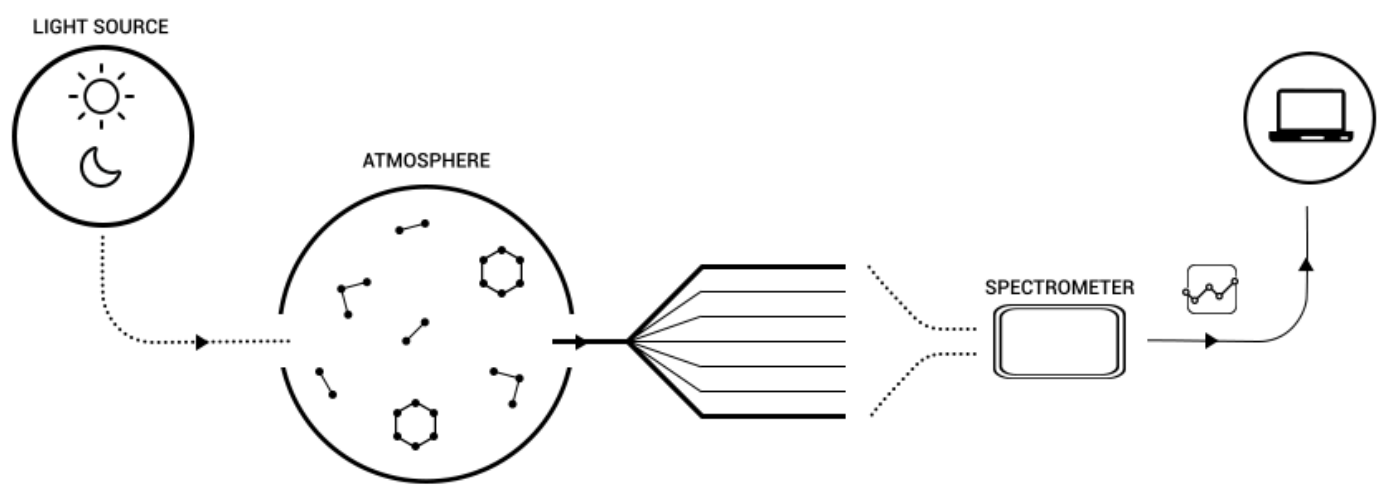

Figure 3. Passive DOAS schematic.

- Mie scattering is caused by particles and larger molecules suspended in the atmosphere and is not very dependent on the wavelength (hence the white colour of clouds).

- Instrumental and turbulence effects are the instrument's transmissivity and atmospheric turbulence in the optical path also limit light intensity.

In addition, we also have to take into account that, in the atmosphere, there are a number of trace gases that interfere with passing light.

Another aspect worth mentioning is that our device is never pointed directly at the light source (the Sun) but always processes light that has been scattered at some unknown point in the optical path. This means that the light that reaches our detector is only the scattered fraction of the sunlight, depending on the system's position and geometry, as well as wavelength.

The expansion of Lambert-Beer's equation to include all these effects results in Eq. (3).

$$
\begin{aligned}
I(\lambda) & =I_{0}(\lambda) \cdot A(\lambda, \ldots) \cdot S(\lambda) \\
& \cdot \exp \left[-\int\left[\left(\sum_{i} \sigma_{i}(\lambda, s) \cdot c_{i}(s)\right)+\epsilon_{\mathrm{M}}(\lambda, s)\right.\right.
\end{aligned}
$$

$$
\left.\left.+\epsilon_{\mathrm{R}}(\lambda, s)\right] \mathrm{d} s\right],
$$

where $A(\lambda, \ldots)$ is the fraction of scattered light that reaches the device, $S(\lambda)$ represents instrumental and turbulence effects, $\sigma_{i}(\lambda, s)$ is the absorption cross section of absorber $i, c_{i}$ is the concentration of absorber $i, \epsilon_{\mathrm{R}}(\lambda)$ represents Rayleigh's extinction coefficient and $\epsilon_{M}(\lambda)$ represents Mie's extinction coefficient.

The interest of this equation lies within the retrieval of $c_{i}$, a given absorber's concentration. Since the integral is taken along the total atmospheric path of the measured photons, and considering that their cross sections do not vary significantly in atmospheric conditions, it is possible to define the concept of slant column, which is of great importance (Merlaud, 2013).

$\mathrm{SC}_{i}=\int c_{i}(s) \mathrm{d} s$

This quantity, as Eq. (4) shows, equals the integral of an individual absorber's concentration along the atmospheric optical path of relevance.

Now, without knowledge of $I_{0}(\lambda)$, these equations cannot give us absolute concentration values. We can, however, use another scattered light spectrum as reference in Eq. (2). Instead of absolute densities, this will yield relative changes in the atmosphere. We thus arrive at Eq. (5). 


$$
\begin{aligned}
\ln \left(\frac{I_{\mathrm{ref}}}{I}(\lambda)\right) & =\ln \left(\frac{A_{\mathrm{ref}}}{A}(\lambda, \ldots)\right)+\ln \left(\frac{S_{\mathrm{ref}}}{S}(\lambda)\right) \\
& +\sum_{i}\left(\sigma_{i}(\lambda) \cdot \Delta \mathrm{SC}_{i}(\lambda)\right)+\Delta \tau_{\mathrm{M}}(\lambda) \\
& +\Delta \tau_{\mathrm{R}}(\lambda),
\end{aligned}
$$

where $\Delta \mathrm{SC}_{i}$ is the relative slant column of absorber $i ; \Delta \tau_{\mathrm{M}}$ is the relative Mie scattering term, integrated to its optical thickness; and $\Delta \tau_{\mathrm{R}}$ is the relative Rayleigh scattering term, integrated to its optical thickness.

This is where the principle of DOAS is applied. Instrument features, scattering and other atmospheric effects have broad absorption spectral profiles, which vary slowly with wavelength. Several trace absorbers have narrow and rapidly varying spectral signatures in at least a small section of the spectrum. By using Eq. (6), we can separate these contributions (Danckaert et al., 2015).

$\sigma(\lambda)=\sigma^{\prime}(\lambda)+\sigma_{0}(\lambda)$

Here, the broad part of the optical thickness $\left(\sigma_{0}(\lambda)\right)$ can be separated from the narrow part $\left(\sigma^{\prime}(\lambda)\right.$ - differential) by approximating it by a low-order polynomial, resulting in Eq. (7).

$\ln \left(\frac{I_{\mathrm{ref}}}{I}(\lambda)\right)=\sum_{i=1}^{n} \sigma_{i}{ }^{\prime}(\lambda) \cdot \Delta \mathrm{SC}_{i}+\sum_{j=0}^{m} a_{j} \cdot \lambda^{j}$

where $\sum_{i=1}^{n} \sigma_{i}{ }^{\prime}(\lambda) \cdot \Delta S C_{i}$ is the differential part (narrowband, rapidly varying with wavelength) and $\sum_{j=0}^{m} a_{j} \cdot \lambda^{j}$ is a low-order polynomial, used to remove the broadband spectral features resulting from atmospheric and instrumental phenomena.

In practice, the mathematical solving of Eq. (7) is not enough since it does not account for the Ring effect or the non-linearities that result from stray light and wavelength shift in measured and cross-section spectra.

The Ring effect is a consequence of rotational Raman scattering: molecules in the atmosphere do not absorb photons in a purely elastic (Rayleigh scattering) fashion. A small portion of the light-matter interaction is in fact inelastic (Brinkmann, 1968; Merlaud, 2013). This changes the light source frequencies as seen from the detector. This phenomenon was first noticed by Grainger and Ring in 1962. At the time, they noticed that the well-known Fraunhofer lines would slightly change when one observed them by using moonlight instead of scattered daylight (Grainger and Ring, 1962).

From the occurrence of these phenomena, it results that the mathematical procedure for DOAS measurements consists in solving a linear and a non-linear problem. The linear problem is solved by writing Eq. (7) in its matrix form:

$\tau=\mathbf{A} \cdot X$.
A is an $m \times n$ matrix, with its columns being the differential cross sections $\sigma_{i}{ }^{\prime}(\lambda)$ and the wavelength powers taking the polynomial $P(\lambda)=\sum_{j=0}^{m} a_{j} \cdot \lambda^{j}$ into account. Since the number of lines in $A$ is much larger than the number of columns, the system is overdetermined and, in this case, we must use methods to numerically approximate a solution. It is common to use the least-squares approach, in which the best solution is the one that minimises $\chi^{2}=$ $[\tau-A \cdot X] \cdot[\tau-A \cdot X]^{T}$.

While the Ring effect is treated as a pseudo-absorber, a synthetically produced (Chance and Spurr, 1997) cross section that is fitted just like any other absorber, non-linearities are addressed by applying Levenberg-Marquardt's approach to non-linear fitting problems to Eq. (9) (Merlaud, 2013; Bevington and Robinson, 2003):

$$
\begin{aligned}
& \ln \left(\frac{I_{\mathrm{ref}}(\lambda)}{I(\lambda+\text { shift })+\text { offset }}\right)=\sum_{i=1}^{n} \sigma_{i}{ }^{\prime}(\lambda) \cdot \Delta \mathrm{SC}_{i} \\
& +\sum_{j=0}^{m} a_{j} \cdot \lambda^{j},
\end{aligned}
$$

where shift and offset, which represent spectral wavelength shifts and stray light offsets, respectively, are responsible for the non-linear character of the problem.

The FFF system and its algorithm are based on the Passive DOAS technique by making scattered sunlight spectral measurements. Our algorithm differs from the original method mainly because of its very particular goal - fire detection through smoke. This objective is very different from the precise quantification of a certain trace gas concentration levels and means that both hardware and software must be adapted to the task at hand, as described in Sect. 4.

\section{The device}

The Forest Fire Finder (see Fig. 4) is a remote sensing system that has the goal of detecting forest fires. It is a sophisticated piece of equipment with many features and customisation possibilities. Its complexity and the fact that it is meant to operate $24 \mathrm{~h}$ per day create a need for control electronics and instrumentation. These devices are out of scope for this paper and will be revisited for another article that will include a detailed description of the FFF control software. This section aims to give a brief and basic hardware/software presentation for daytime spectroscopic operation and fire detection.

The FFF scans the horizon for the presence of a column of smoke by performing sequential spectroscopic measurements of its surrounding environment using only the Sun as a light source, as illustrated by Fig. 5. Sunlight is captured with a Maksutov-Cassegrain telescope and guided through an optical fiber cable into a spectrometer, which will transform it into an electric signal.

The system has to cover wide areas, which is why the telescope is mounted on an ENEO VPT-501 pan and tilt unit 


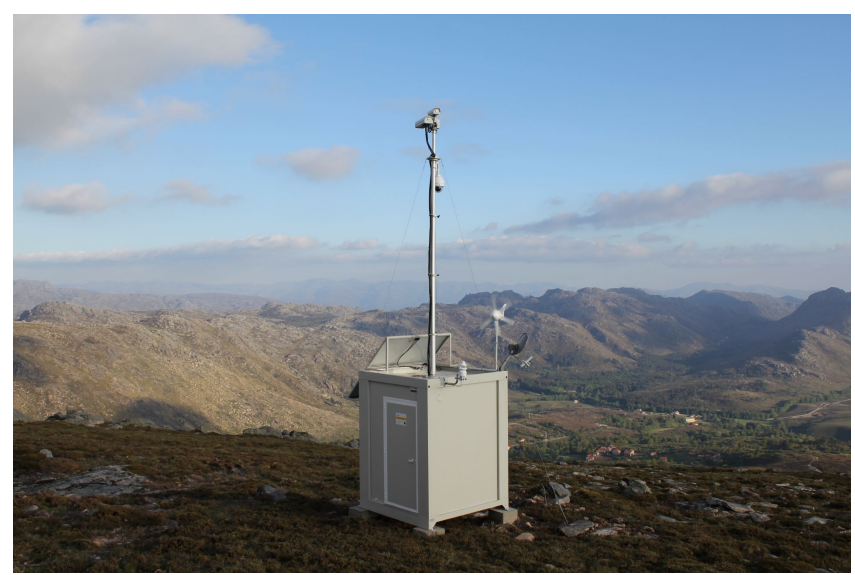

Figure 4. The Forest Fire Finder system in one of its deployment locations in the north of Portugal.

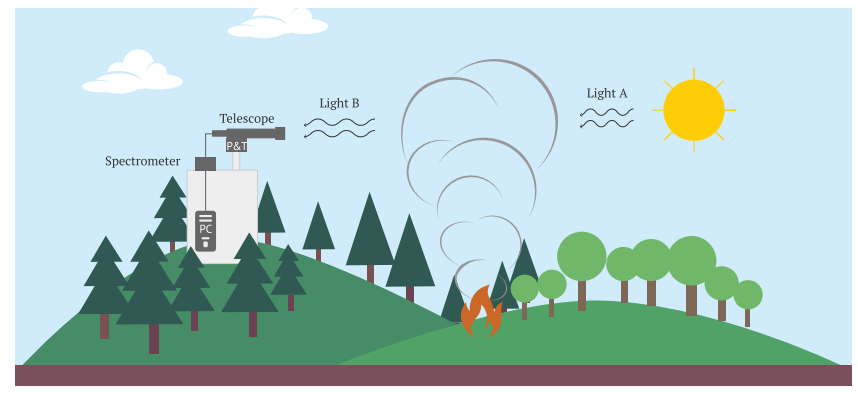

Figure 5. FFF illustration. The system continuously scans the horizon, in search of a smoke column.

that ensures the device's movement. The pan and tilt head unit assembly also includes a full-HD camera, which is used primarily for the optical alignment of the system and for human validation and supervision. During the night, this camera is also used for fire detection purposes; however, that is not within the scope of this paper and will be approached in another publication.

The Maksutov-Cassegrain telescope design uses the folded tube of the Cassegrain types and the spherical shape of primary mirror, secondary mirror and corrector lens of the Maksutov. In the FFF case, the chosen $90 \mathrm{~mm}$ aperture and $13.8 f$ ratio telescope, with a field of view of $1.4^{\circ}$, represents the best compromise between size, magnification and amount of captured light. In addition, it is also a costeffective solution for the task at hand.

The AvaSpec 2048 is a popular 2048-pixel CCD photo array spectrometer. It can be customised with several slit sizes and gratings in order to suit the application it is intended for. In the case of the FFF system, a $50 \mu \mathrm{m}$ slit is used in conjunction with a 300 lines $\mathrm{mm}^{-1}$ grating, which ensures a wavelength range of $800 \mathrm{~nm}$, from 300 to $1100 \mathrm{~nm}$ at a spectral resolution of $2.4 \mathrm{~nm}$.
The spectrometer is connected to a computer, which is responsible for data processing and fire detection. It runs a custom-made software, developed in MathWorks' MATLAB development suite and C\#. This software is deployed as a Microsoft Windows Service, as part of the FFF software suite.

\section{Automatic smoke detection}

The Forest Fire Finder is an electronic device that performs a spectroscopic analysis of the sky above the horizon, with the aim of detecting the presence of a smoke column. Smoke detection depends on the fire's emissions, which influence the composition of the atmosphere and on the system's spectroscopic algorithms, which allow those changes to be detected.

\subsection{Forest fire emissions and DOAS}

Forest fire smoke is a complex mixture of gases and aerosols that considerably changes the atmosphere (Urbanski et al., 2008). Among its key components are carbon oxides $\left(\mathrm{CO}\right.$ and $\left.\mathrm{CO}_{2}\right)$, methane $\left(\mathrm{CH}_{4}\right)$, non-methane hydrocarbons, volatile organic components, nitrous oxides $\left(\mathrm{NO}_{x}\right)$ and particulate matter (Van Der Werf et al., 2010; Ward and Hardy, 1991; Spichtinger et al., 2004). Trace gases in smoke have a definite impact on the atmosphere's optical properties since some absorb light in the visible region of the electromagnetic spectrum. In addition to this, and depending on the combustion process, fire gives rise to the formation and emission of solid particles (Ward and Hardy, 1991). Given their size, these particles become aerosols, which influence light in all wavelengths due to Mie's scattering.

In contrast, fire emissions also alter the balance between the perceived column densities of water $\left(\mathrm{H}_{2} \mathrm{O}\right)$, oxygen $\left(\mathrm{O}_{2}\right)$, ozone $\left(\mathrm{O}_{3}\right)$ and the oxygen dimer $\left(\mathrm{O}_{4}\right)$. All of these chemicals' cross sections are significant in the visible part of the spectrum.

Passive DOAS measurements are commonly used to retrieve the atmospheric column densities of several chemical compounds. Smoke columns, however, present themselves as sudden and localised changes in atmospheric concentrations. If one were to use this technique and analyse their absolute concentration values per se, it would be very difficult to infer the presence of smoke.

This does not mean passive DOAS cannot be used in this context. In fact, this method is very effective in detecting smoke if we put a "smoky" spectrum as $I$ and a "normal" spectrum as $I_{0}$ in Eq. (2), resulting in a signal as displayed in Fig. (7). Thus, by continuously acquiring spectra in a set of fixed azimuths and comparing the retrieved DOAS signals in pairs by azimuth, a narrow change such as the one produced by a forest fire becomes discernible in time. The FFF algorithm, presented in Sect. 5.2, does precisely this. These alterations are often difficult for the human eye to see, but there are some artificial intelligence algorithms that 


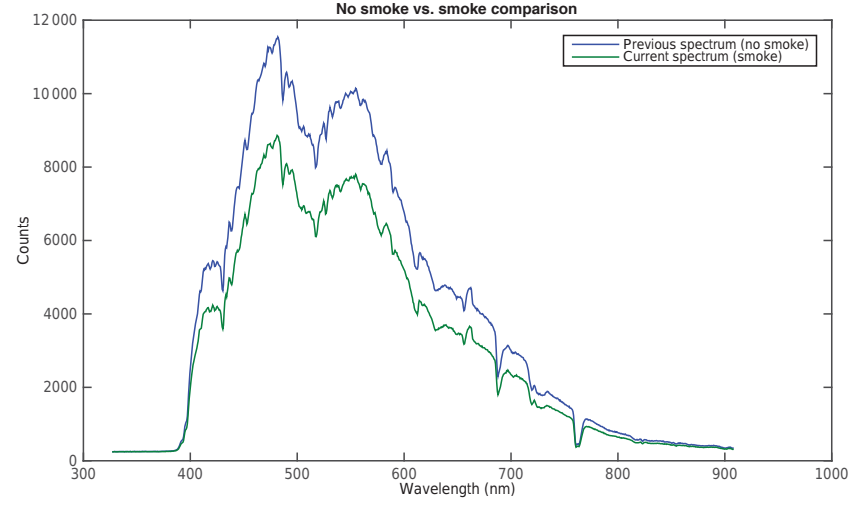

Figure 6. This plot shows how a smoke column can influence a spectral measurement. Both these spectra were acquired on 29 December 2014, with a time difference of approximately $5 \mathrm{~min}$ and no azimuth difference.

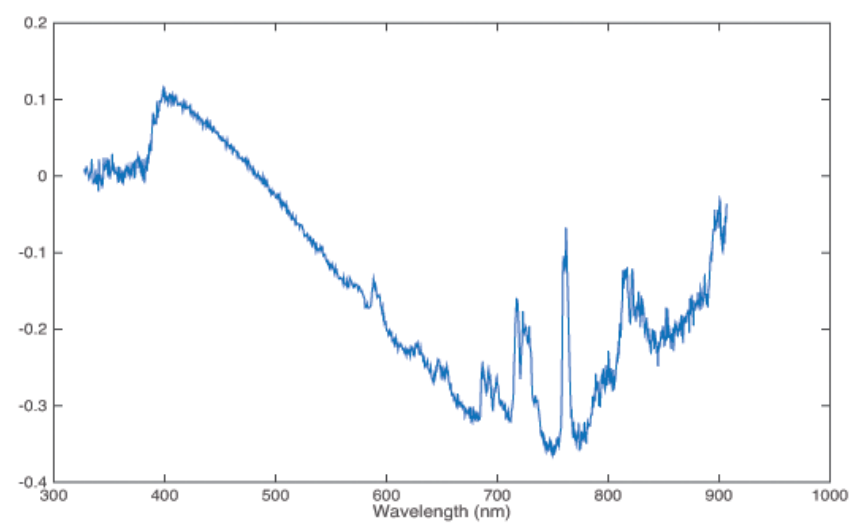

Figure 7. Example of a signal obtained by dividing two consecutive spectra of the same azimuth and calculating the logarithm of this division, the differential optical thickness. It is this signal that is fitted through Eq. (9).

have been shown to be effective in separating the sky from a smoke column event and which we will discuss in Sect. 5.2.2.

\subsection{The FFF algorithm}

The FFF algorithm uses the mathematical ingenuity behind DOAS measurements to attempt the detection of forest fire smoke columns. It is important to bear in mind that this device is meant for real-time automatic detection of a forest fire. This creates strict time and memory constraints that the algorithm must adapt to in order to accomplish its function.

The system relies on its continuous movement at constant speed to provide spatially accurate detections. The spectrometer acquires 2 spectra $\mathrm{s}^{-1}$, which are all analysed by the computer. Spectral integration time varies from $60 \mathrm{~ms}$ to $450 \mathrm{~ms}$ and is typically $210 \mathrm{~ms}$. DOAS calculations are currently taking between $250 \mathrm{~ms}$ and $350 \mathrm{~ms}$ in the industrial computers powered by Intel i5 we use on the system. This means that the software is almost always lagging behind the hardware. The system is designed to cope with this delay, and does so with great robustness, but in larger scans this lag becomes sufficiently significant as to compromise real-time fire detection.

These limits are reflected in several steps in the DOAS calculation process, such as the non-inclusion of stretch effects in the non-linear DOAS problem or the consideration that there are no atmospheric temperature fluctuations over the optical path. This approach would not be valid if we wanted to make a precise quantification of a certain compound's atmospheric column, but it works given the fact that we only want to distinguish between a smoky spectrum and non-smoky spectrum.

The algorithm is divided into two separate phases. The first phase, which is run in real time as soon as the spectrum arrives, is the chemical phase, described in Sect. 5.2.1. Results from this stage are stored in memory and accessed at the end of each scan by the second phase, which performs the classification of the analysis as a detection or a non-detection. This phase is described in Sect. 5.2.2.

\subsubsection{The chemical phase}

This algorithm section happens immediately after spectral acquisition. It corresponds to a passive DOAS analysis (see Sect. 3) of the spectrum in two different conceptual levels, as illustrated in Fig. 10. The first level uses the same azimuth spectrum of the previous scan as a reference spectrum in the DOAS calculations. The second level uses the mean of the ten spectra immediately to the left and to the right of the analysed spectrum for the same purpose. This processing level was created in an empirical way, after observing that in the presence of strong winds, smoke columns move horizontally. Both levels are calculated using literature spectra, compiled in Table 1. In practice, these two processing levels represents three possible ways of applying Eq. (2): considering $I_{0}$ as the previous spectra acquired with the same azimuth; considering $I_{0}$ as the average of the ten immediate spectra to the left of current azimuth; and considering $I_{0}$ as the average of the ten immediate spectra to the right of current azimuth (this last method implies delayed processing of the current spectrum).

In this stage, the algorithm proceeds to retrieve column density values for $\mathrm{NO}_{2}, \mathrm{H}_{2} \mathrm{O}, \mathrm{O}_{2}, \mathrm{O}_{3}$ and $\mathrm{O}_{4}$, using Eq. (9) and the Levenberg-Marquardt algorithm. Figures 8 and 9 show actual retrieved column densities for one of the PenedaGerês FFF systems, taken on 26 December 2014. The charts were produced using the QDOAS software (Danckaert et al., 2015). These plots are all relative to the first level of processing, as described above. In parallel to the column density retrieval, short- and long-wavelength energy contribution and signal-to-noise ratio (SNR) are also computed for the analysed spectrum optical density (with relation to the previous spectrum of the same azimuth). All the processed data are 
stored in a single matrix, which will be used by the second stage of the FFF algorithm, described in Sect. 5.2.2.

As stated, in the first level of processing the FFF uses the same azimuth spectrum of the previous scan as a reference. This means that between the spectrum of interest and the reference there is only an approximately $5 \mathrm{~min}$ difference. This in turn implies that, when plotted, the fitting signal is almost always negligible for non-smoky spectra (see the top of Figs. 8 and 9). However, smoky spectra have higher column density differences, which produce fitting plots with somewhat more-pronounced signals (see bottom of Figs. 8 and 9).

At this point, it is important to note two things. First and foremost, this kind of measurements produce results that are near the limit of the FFF's detection capabilities, which can still be numerically used to train a detection algorithm. Second, it should be highlighted that smoke-detection patterns were not manually defined. It would be extremely hard for a human to consider all the subtle changes in the millions of spectra acquired by the FFF systems and find a discerning pattern with that information. Instead, and as will be shown in Sect. 5.2.2, a machine learning algorithm was used to perform this task.

\subsubsection{The classification phase}

The classification stage runs at the end of each scan and goes through the chemical data gathered and stored in the previous algorithmic stage. It is divided into two levels, which are sequentially run. The first level acts as a filter for the second level, which is comprised of a support vector machine (SVM) classifier, an artificial intelligence supervised learning algorithm.

The first level starts by assembling two signals from the gathered data: one is comprised of the SNR value for each spectrum in the scan and the other is assembled by calculating an average signal energy per pixel, by dividing the sum of the square of each spectrum and by the number of its pixels and sequentially storing this result in an array.

The algorithm then applies a peak detection routine to the second artificial signal. For each detected peak, the system evaluates the SNR of the corresponding spectrum and compares it to a fixed threshold value, which is set in a configuration file. If this value is higher than said threshold, the spectrum is marked to be further analysed.

The next step involves the calculation of column density ratios between $\mathrm{O}_{2}, \mathrm{O}_{4}, \mathrm{NO}_{2}, \mathrm{H}_{2} \mathrm{O}$ and $\mathrm{O}_{3}$ for the marked spectra. These ratios are then fed to the SVM, which returns a binary classification result (1 or 0$)$.

An SVM is an algorithmic approach to the problem of classification in the context of supervised learning (Press et al., 2007). Introduced in 1992, by Boser et al. (1992), this method has since proved itself of great usefulness by providing relatively straightforward solutions to previously complicated classification applications. SVMs are generally easier to implement and understand, and this has also contributed
Table 1. Literature spectra used for the FFF's passive DOAS calculations. All cross sections are downloaded from the MPI-Mainz UV/VIS Spectral Atlas of Gaseous Molecules of Atmospheric Interest (Keller-Rudek et al., 2013).

\begin{tabular}{lrrr}
\hline Compound & $\begin{array}{r}\text { Interval used } \\
(\mathrm{nm})\end{array}$ & Reference key & Year \\
& $600-800$ & Bogumil et al. (2003) & 2003 \\
\hline Oxygen $\left(\mathrm{O}_{2}\right)$ & $500-650$ & Bogumil et al. (2003) & 2003 \\
Ozone $\left(\mathrm{O}_{3}\right)$ & $400-800$ & Hermans (2011) & 2011 \\
Oxygen dimmer $\left(\mathrm{O}_{4}\right)$ & $400-500$ & Coheur et al. (2002) & 2002 \\
Water vapour $\left(\mathrm{H}_{2} \mathrm{O}\right)$ & $400-500$ & Vandaele (2002) & 2002 \\
Nitrogen dioxide $\left(\mathrm{NO}_{2}\right)$ & &
\end{tabular}

for their fast spread. The general concept behind the SVM methodology is to find and define the hyperplane that better separates data into two classes (Press et al., 2007).

Like all supervised learning techniques, SVMs need to be trained prior to being used. In the case of the FFF classification algorithm, SVMs were trained in successive generations, with each generation built upon the results of the previous.

The first FFF SVM generation was built using data from 60 different moments in 2014 (fire and non-fire), in 13 different locations in the Peneda-Gerês National Park (PNPG). A specially designed software tool was used to manually search and store fire data from 30 different events and 30 non-fire moments in said year and an SVM was trained with the resulting information. Another custom-made software was used to classify data of several months with the created SVM. The second-generation SVM was created by repeating the process, taking the results of the first-generation SVM into account and testing against a new set of data.

One downside of using this kind of classifier is that, after training and application, the classification becomes opaque. It is not feasible to understand what caused a false detection. This raises the problem of how to improve the detection capabilities of the FFF system. There are two fundamental approaches to this issue: focusing on the SVM or adding more information to the decision process.

The current line of thought concerning this situation is that, in normal circumstances, the classifier's performance is quite satisfactory. Still, some peculiar events cause the system to issue wrong detections. These events are many times of systematic nature, such as particular kind of cloud that only appears in the early morning or emissions from local industrial facilities. These interferences can be detected themselves, without altering the smoke column detection algorithm, which is where the majority of improvement efforts will be made in the near future (see Sect. 7).

Finally the algorithm reaches the final point, at which it has to say whether the data that were run correspond to a smoke column in the horizon. The spectral information entered to the SVM includes the results from the two chemical processing levels debated in Sect. 5.2.1 and originates three classification possibilities: 

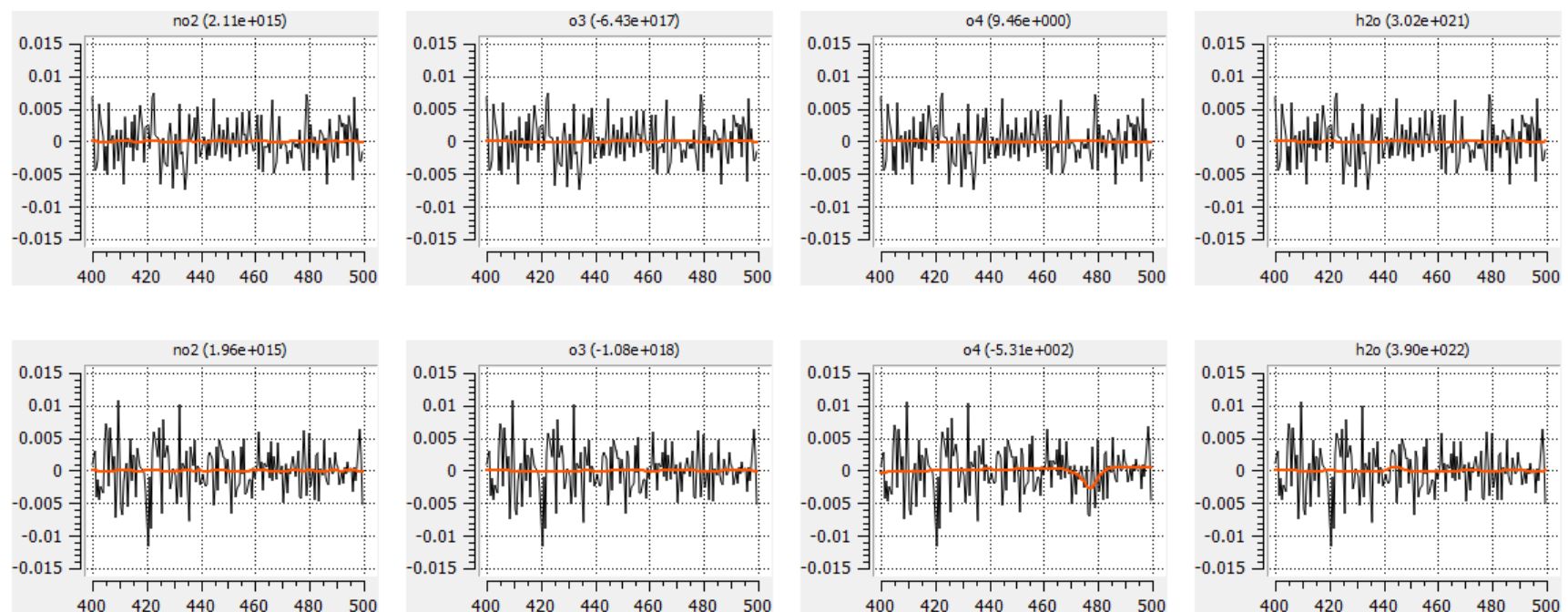

Figure 8. Column densities for $\mathrm{NO}_{2}, \mathrm{O}_{3}, \mathrm{O}_{4}$ and $\mathrm{H}_{2} \mathrm{O}$, retrieved between 400 and $500 \mathrm{~nm}$. The plots below correspond to a smoke column detection. In these plots, the horizontal axis corresponds to wavelength in $\mathrm{nm}$ and the vertical axis to the differential optical thickness.
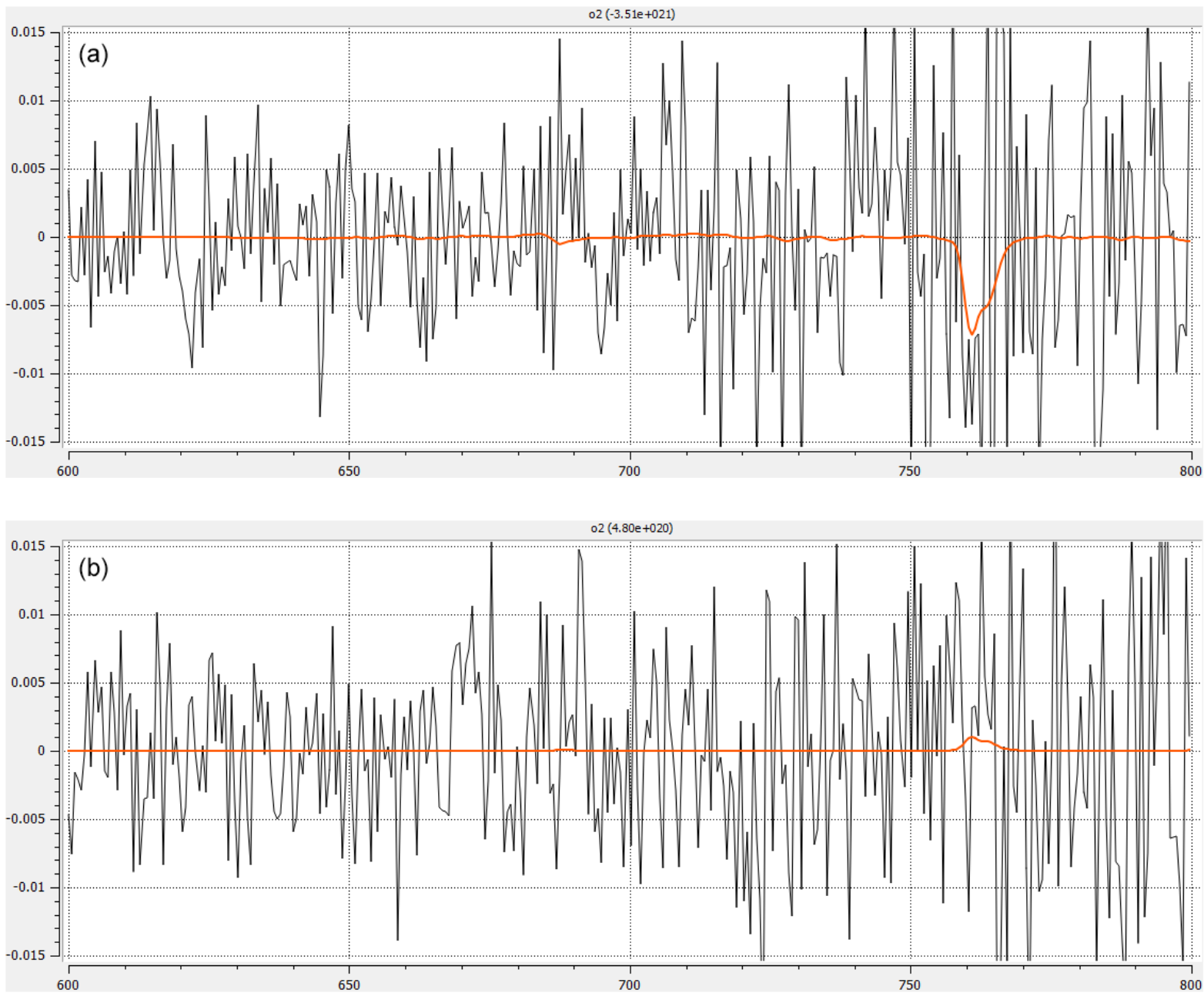

Figure 9. Column density for $\mathrm{O}_{2}$, retrieved between 600 and $800 \mathrm{~nm}$. The plot below corresponds to a smoke column detection. In these plots, the horizontal axis corresponds to wavelength in $\mathrm{nm}$ and the vertical axis to the differential optical thickness. 

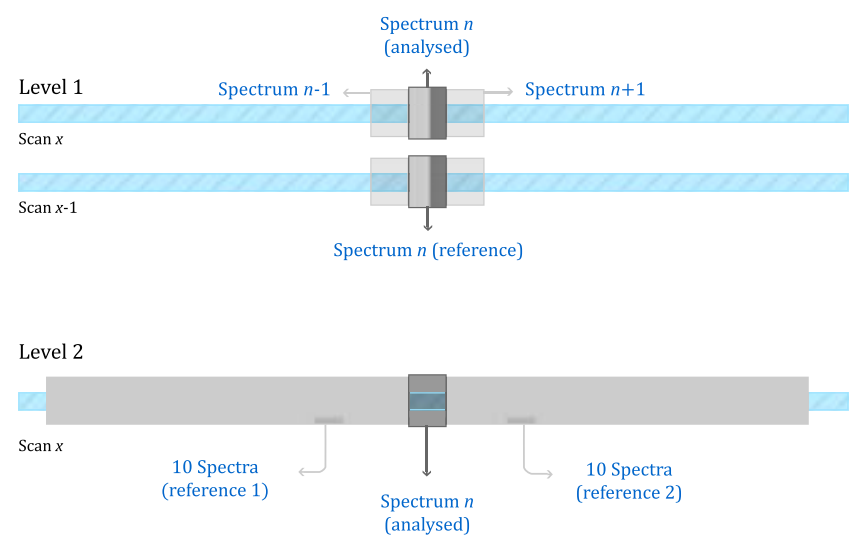

Figure 10. Illustration of FFF's two processing levels in the chemical stage of the algorithm.

- smoke column detected between previous and current scan;

- smoke column detected on the left of analysed spectrum;

- smoke column detected on the right of analysed spectrum.

If two of these classification results are positive, the system issues an alarm.

\section{Results and discussion}

In 2013, 13 FFF devices were deployed in the PNPG, in the north of Portugal. Their placement reflected topography, local accessibility and fire protection needs.

In 2015, FFF data were gathered and compared to official data from the Portuguese National Authority of Civil Protection (ANPC), the country's institution responsible for forest fire protection and management.

Table 2 shows said data and comparison. In it, a confirmed detection occurs when a smoke column is sensed by an FFF device and the detection is validated by a human operator. This is different from a registered fire event (RFE), which is a fire that was inserted into ANPC's database.

Official statistics count 132 fire events in 2015 within PNPG. During the same period, the FFF network issued 578 detections, of which 369 were false detections, and 209 confirmed events, of which 53 were coincident with RFEs.

Although the false detections may seem to be high in comparison to confirmed detection, it is important to bear in mind that each system has an average working period of $12 \mathrm{~h}$ per day. At two spectra per second, this means an average of 86400 analysed spectra per system per day. Since each and every one of these analysis can trigger an alarm by itself, false detections reach only $0.0000833 \%$ in all systems. In addition, there have been some events that were wrongly
Table 2. FFF statistics for 2015 in the Peneda-Gerês National Park.

\begin{tabular}{lr}
\hline Registered fire events & 132 \\
Total fire detections & 578 \\
False events & 369 \\
Confirmed detections & 209 \\
Estimated network & 56940 \\
Operation time (h) & \\
Estimated analysis & 409968000 \\
False detection \% & 0.0000900 \\
False alarms per system/day & 0.07776607 \\
\hline
\end{tabular}

marked as false detections due to misunderstandings on how the system is to be handled by humans. These events correspond mainly to small fires and prescribed burns, large enough to be detected. Security issues, regarding the Portuguese Civil Protection Authority, prevent the exact quantification of human errors, but they amount to a significant percentage of false alarms.

Although the presented numbers are enough to paint a general picture of the FFF system's behaviour, the available data do not allow a thorough quantification of the system's performance, since there is no correct gold standard regarding forest fires due to fire registry procedures not being clearly established. This becomes exceedingly apparent when comparing the number of RFEs and the number of confirmed detections: every confirmed detection was deemed relevant by a human operator, yet there are only 132 RFEs for the 209 confirmed detections.

Another important result that becomes noticeable in Table 2 is the fact that false positives and true detections vary in similar ways. This can be explained by the fact that the FFF is a spectroscopic system at its heart. Fire releases chemical components into the atmosphere, which in turn are detected by the system. If there are many fires in a small geographic region, such as the PNPG, it is possible that an FFF is able to sense it, without the presence of a visible smoke column in its patrol path. We have also noticed a trend for false alarms on specific cloudy days. We believe this is due to pollutant particles carried by the clouds. Light scattering by these particular clouds sometimes breaches through the system's energy and SNR filters (see Sect. 5.2.1) and is incorrectly classified as a smoke column.

\section{Future developments}

The FFF has been an ongoing development for NGNS-IS, Ltd. Since 2006, the device has undergone two complete redesign processes, motivated by hardware improvements. The current version is without a doubt the most robust and reliable design ever, achieving uptimes of $99 \%$. There will always be room for improvement regarding hardware, but given the operating level of the current version these will not be a priority in the near future. 


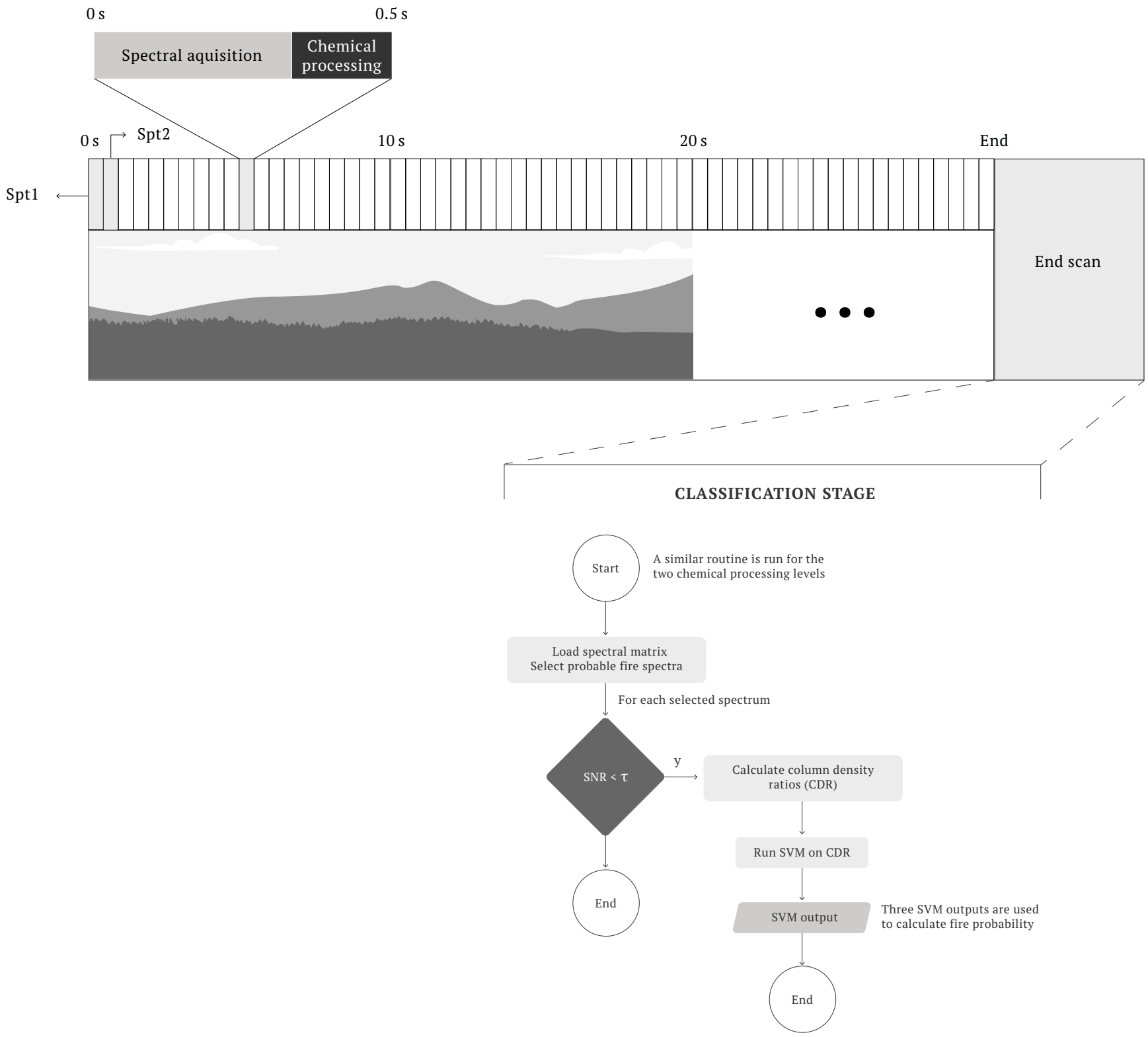

Figure 11. FFF algorithm simplified work-flow diagram.

The software architecture selected for the system allows total freedom for future development needs, with minimal integration efforts. As stated in Sect. 4, this was a requirement because most customers need some level of customisation.

As mentioned in Sect. 5, the FFF is subject to a number of strict time constraints, mostly related to the real-time nature of the system. As a consequence, one of our ever-present goals is algorithm optimisation. Currently, the spectral algorithms are being rewritten, with the inclusion of more features in less processing time. It is also true that as computers become more powerful, it is possible to add more details to the algorithms.
Another line of research currently being pursued is the study of false positives and their relation to the presence of aerosols in the atmosphere. These developments are, however, entirely dependent on the optimisation, since it is currently not possible to add this functionality without sacrificing real-time operation.

DOAS (see Sect. 3) is a widely used atmospheric analysis technique, with much broader uses than just fire sensing. In fact, the technique is used for urban air pollution monitoring (OPSIS, 2016), almost-real-time volcanic plumes monitoring for aviation control (Brenot et al., 2014) and quantification of volcanic gases (Galle et al., 2010). The experience attained while developing FFF allowed the creation of another 
project, called Project ATMOS, designed to monitor and control air pollution, crop maturity, water stress and agricultural plagues. This project started in April 2016 and is expected to conclude in September 2019.

\section{Conclusions}

Life on Earth is greatly influenced and shaped by fire events. Humans in particular depend on fire to maintain their technology and way of life. However, forest fires are a global menace that cause concern all over the world. Several industrialised countries have allocated a great deal of resources to researching wildfires and their behavioural dynamics.

It is this concern that generates a very large market for remote sensing equipment for early forest fire detection, a market which is expected to grow $11.53 \%$ by 2020 .

This article addresses one of such equipments. The FFF was developed in Lisbon in 2006 by NGNS-IS, Ltd. and is the only one that is based on optical spectroscopy, particularly differential optical absorption spectroscopy.

In 2013, a 14-element network of FFF devices was installed in the Peneda-Gerês National Park. In 2015, this network was able to detect a confirmed 209 fire events, a number significantly higher than the officially registered 132 fire occurrences in the same region.

For the same period the system has issued 369 false detections, but it is worth considering that confirmed and false detections have similar trends. This is due to the fact that fires pollute the atmosphere with the chemicals that the system aims to detect and is in agreement with the device's operating physical principle, optical spectroscopy.

Evaluation of a fire detection system is a very difficult task. There is no formal definition of how large a forest fire must be to be considered an event and this means there is no perfect classification model (a gold standard) to compare the system's performance to.

The FFF is an automatic forest fire detection system which has proven itself to be effective and detected a great number of forest fires (see Table 2 in Sect. 6). In addition, the system's current hardware and software configuration has resulted in extremely high uptime levels, contributing for an adequate fire detection coverage and, consequently, optimal levels of fire protection.

Data availability. The data used in this paper are not publicly available nor are we authorised to make them so.

Competing interests. The authors are affiliated with NGNS, which developed the Forest Fire Finder system and may be affected by the research reported in the enclosed paper.
Acknowledgements. The authors would like to thank the NGNS team for their support, especially Hélia Pinto, for the numerous drawings and diagrams. This work was funded in part by the FCT PhD grant PD/BDE/114549/2016 and by the FEDER3491 project, through Lisbon's Operational Program LISBOA2020.

Edited by: M. Van Roozendael

Reviewed by: two anonymous referees

\section{References}

Alkhatib, A. A. A.: A review on forest fire detection techniques, International Journal of Distributed Sensor Networks, 2014, 597368, https://doi.org/10.1155/2014/597368, 2014.

Bevington, P. R. and Robinson, D. K.: Data Reduction and Error Analysis for the Physical Sciences, 2003.

BNHCRC: Bushfire \& Natural Hazards CRC, available at: http:// www.bnhcrc.com.au/home, last access: 10 May 2016.

Bogumil, K., Orphal, J., Homann, T., Voigt, S., Spietz, P., Fleischmann, O., Vogel, A., Hartmann, M., Kromminga, H., Bovensmann, H., Frerick, J., and Burrows, J.: Measurements of molecular absorption spectra with the SCIAMACHY pre-flight model: instrument characterization and reference data for atmospheric remote-sensing in the $230-2380 \mathrm{~nm}$ region, J. Photoch. Photobio. A, 157, 167-184, https://doi.org/10.1016/S10106030(03)00062-5, 2003.

Boser, B. E., Guyon, I. M., and Vapnik, V. N.: A training algorithm for optimal margin classifiers, in: Proceedings of the fifth annual workshop on Computational learning theory COLT '92, ACM Press, New York, New York, USA, 144-152, https://doi.org/10.1145/130385.130401, 1992.

Brenot, H., Theys, N., Clarisse, L., van Geffen, J., van Gent, J., Van Roozendael, M., van der A, R., Hurtmans, D., Coheur, P.-F., Clerbaux, C., Valks, P., Hedelt, P., Prata, F., Rasson, O., Sievers, K., and Zehner, C.: Support to Aviation Control Service (SACS): an online service for near-real-time satellite monitoring of volcanic plumes, Nat. Hazards Earth Syst. Sci., 14, 1099-1123, https://doi.org/10.5194/nhess-14-1099-2014, 2014.

Brinkmann, R. T.: Rotational Raman scattering in planetary atmospheres, Astrophys J., 154, 1087-1093, https://doi.org/10.1086/149827, 1968.

Chance, K. V. and Spurr, R. J. D.: Ring effect studies: Rayleigh scattering, including molecular parameters for rotational Raman scattering, and the Fraunhofer spectrum, Appl. Opt., 36, 5224, https://doi.org/10.1364/AO.36.005224, 1997.

Coheur, P.-F., Fally, S., Carleer, M., Clerbaux, C., Colin, R., Jenouvrier, A., Mérienne, M.-F., Hermans, C., and Vandaele, A. C.: New water vapor line parameters in the 26000-region, J. Quant. Spectrosc. Ra., 74, 493-510, https://doi.org/10.1016/S00224073(01)00269-2, 2002.

Danckaert, T., Fayt, C., Van Roozendael, M., de Smedt, I., Letocart, V., Merlaud, A., and Pinardi, G.: QDOAS, available at: http: //uv-vis.aeronomie.be/software/QDOAS/ (last access: 12 June 2017), 2015.

Envirovision Solutions: Forestwatch by EnviroVision Solutions, EVS, 4th Wildfire Detection Workshop, 11-12 March 2015, Edmonton, Alberta, Canada, 2015. 
European Comission: Catalogue of EU funded projects in Environmental research, 0, 2012.

Food and Agriculture Organisation (FAO): Fire management global assessment 2006, FAO Forestry Paper 151, 135 pp., available at: http://www.fao.org/docrep/009/a0969e/a0969e00. htm (last access: 12 June 2017), 2007.

Galle, B., Johansson, M., Rivera, C., Zhang, Y., Kihlman, M., Kern, C., Lehmann, T., Platt, U., Arellano, S., and Hidalgo, S.: Network for Observation of Volcanic and Atmospheric Change (NOVAC) - A global network for volcanic gas monitoring: Network layout and instrument description, J. Geophys. Res.-Atmos., 115, 1-19, https://doi.org/10.1029/2009JD011823, 2010.

Grainger, J. F. and Ring, J.: Anomalous Fraunhofer Line Profiles, Nature, 193, 762-762, https://doi.org/10.1038/193762a0, 1962.

Hermans, C.: Collisionally induced absorption spectrum of oxygen attributed to the oxygen dimer (O2)2, Tech. rep., BIRA-IASB Spectrocscopy Lab, available at: http://spectrolab.aeronomie.be/ index.htm (last access: 12 June 2017), 2011.

Hough, G.: Vision Systems for Wide Area Surveillance: ForestWatch - a long-range outdoor wildfire detection system, in: TASSIE FIRE Conference Proceedings, https://doi.org/10.1017/CBO9781107415324.004, 2007.

IPCC: Managing the risks of extreme events and disasters to advance climate change adaptation, https://doi.org/10.1596/978-08213-8845-7, 2012.

IQ-Wireless: FireWatch, available at: http://www.fire-watch.de/, last access: 19 May 2016.

Keller-Rudek, H., Moortgat, G. K., Sander, R., and S“rensen, R.: The MPI-Mainz UV/VIS Spectral Atlas of Gaseous Molecules of Atmospheric Interest, Earth Syst. Sci. Data, 5, 365-373, https://doi.org/10.5194/essd-5-365-2013, 2013.

Liyang, Y., Wang, N., and Meng, X.: Real-time forest fire detection with wireless sensor networks, in: Proceedings, 2005 International Conference on Wireless Communications, Networking and Mobile Computing, 2, 1214-1217, https://doi.org/10.1109/WCNM.2005.1544272, 2005.

Manyangadze, T.: Forest fire detection for near real-timemonitoring using geostationary satellites, International Institute for GeoInformation Science and Earth Observation, MSc, 80, 2009.

Merlaud, A.: Development and use of compact instruments for tropospheric investigations based on optical spectroscopy from mobile platforms, Books.Google.Com, 2013.

NGNS-IS, L.: Long range fire detection exclusive solution, available at: http://www.ngns-is.com/products/ngns-all-over/ forest-fire-finder, last access: 10 May 2016.
OPSIS: Opsis, availabel at: http://www.opsis.se/Aboutus/tabid/996/ Default.aspx, last access: 19 May 2016.

Platt, U. and Stutz, J.: Differential Optical Absorption Spectroscopy, Springer, Heidelberg, Germany, 2007.

Press, W. H., Teukolsky, S. A., Vetterling, W. T., and Flannery, B. P. Numerical Recipes: The Art of Scientific Computing, 3rd Edn., 2007.

Research and Markets: Fire Protection Systems Market by Technology (Active, Passive), Product (Fire Detection (Sensors \& Detectors, RFID), Management (Fire Sprinklers, Extinguishers), Fire Analysis \& Response), Service, Vertical, and Geography - Global Forecast to 2022, Tech. rep., Research And Markets, avaible at: http://www.researchandmarkets.com/reports/ 3696148/fire-protection-systems-market-by-technology (last access: 12 June 2017), 2016.

Spichtinger, N., Damoah, R., Eckhardt, S., Forster, C., James, P., Beirle, S., Marbach, T., Wagner, T., Novelli, P. C., and Stohl, A.: Boreal forest fires in 1997 and 1998: a seasonal comparison using transport model simulations and measurement data, Atmos. Chem. Phys., 4, 1857-1868, https://doi.org/10.5194/acp-4-18572004, 2004.

United States Forest Department: The Rising Cost of Fire Operations: Effects on the Forest Service's Non-Fire Work, United States Department of Agriculture, 13 pp., available at: http://www.fs.fed.us/sites/default/files/media/2014/34/ nr-firecostimpact-082014.pdf (last access: 12 June 2017), 2015.

Urbanski, S. P., Hao, W. M., and Baker, S.: Chapter 4 Chemical Composition of Wildland Fire Emissions, Develop. Environ. Sci., 8, 79-107, https://doi.org/10.1016/S1474-8177(08)00004$1,2008$.

van der Werf, G. R., Randerson, J. T., Giglio, L., Collatz, G. J., Mu, M., Kasibhatla, P. S., Morton, D. C., DeFries, R. S., Jin, Y., and van Leeuwen, T. T.: Global fire emissions and the contribution of deforestation, savanna, forest, agricultural, and peat fires (1997-2009), Atmos. Chem. Phys., 10, 11707-11735, https://doi.org/10.5194/acp-10-11707-2010, 2010.

Vandaele, A. C.: High-resolution Fourier transform measurement of the NO 2 visible and near-infrared absorption cross sections: Temperature and pressure effects, J. Geophys. Res., 107, 4348 , https://doi.org/10.1029/2001JD000971, 2002.

Ward, D. E. and Hardy, C. C.: Smoke emissions from wildland fires, Environment International, 17, 117-134, https://doi.org/10.1016/0160-4120(91)90095-8, 1991. 